\title{
Editorial
}

\section{Unique Identifiers for Authors}

\author{
(D)John H.R. Maunsell \\ Editor-in-Chief
}

Scientific productivity is most often measured by the number and quality of published articles. Publishing solid, new, and interesting findings is critical for career advancement. Given the central role of publications in science, it is remarkable that we have tolerated the imprecision of using authors' names to locate their contributions. Few scientists are uniquely identified by their family name and initials, and locating work from individuals with common names is generally a nuisance and sometimes a challenge.

A comprehensive solution is finally available. The Journal of Neuroscience is pleased to announce its participation in the ORCID community. ORCID is an open, non-profit, communitybased effort to create a permanent registry of unique identifiers that will provide a robust way to link publications and other types of scientific contributions to specific individuals. ORCID identifiers will ensure that credit is assigned to the appropriate person.

Authors submitting manuscripts to The Journal will be invited to add their ORCID identifier (if they have one) or to be redirected to the ORCID site (http://orcid.org) to register. Once an author provides their ORCID identifier, it will be tied to their profile and automatically available for future submissions. When an identifier has been provided, a link to the author's ORCID information will appear in published articles, as on this editorial. For more information about ORCID, please go to http://orcid.org.

Unique author identifiers could have far-reaching effects. Although identifiers will be embedded only in future publications, it will be possible for existing online indices of publications to add author identifiers for published articles, making it possible to unambiguously locate all the works of a given investigator. Fully deployed in this way, author identifiers will greatly facilitate locating all the works of individuals and assessing the output of individuals, departments, institutions, and programs. We encourage all our authors to take advantage of this new mechanism for laying claim to their research contributions. 